\title{
Global Minimization of Vertex Height Differences for Freeform Architectural Design
}

\author{
Simon Kulovec, Leon Kos, Jože Duhovnik
}

Faculty of Mechanical Engineering, University of Ljubljana, Ljubljana, Slovenia.

Email: simon.kulovec@lecad.si

Received June $15^{\text {th }}, 2012$; revised July $20^{\text {th }}, 2012$; accepted August $3^{\text {rd }}, 2012$

\begin{abstract}
Architectural design is leading us in the direction of structures with free and irregular forms. As a consequence of this the connection between the design's intent and its fabrication represents a challenge when creating a support structure that is geometrically viable and which needs to possess certain aesthetic, fabricational, thermal and strength requirements. To ensure the contacts of the edges of the neighboring insulation panels along their thicknesses, the edges must be cut at different angles, which causes differences in the vertex heights and, furthermore, differences in the positions of the inner metal sheets of the insulation panels. The main goal of the presented research is the development of a post-optimization procedure, by which the minimum joint-height differences will be achieved for all the joints, taking into account all the free-form surfaces of the individual architectural design. To compensate for the residual height differences the use of spacers of different thicknesses is proposed. The paper considers the global minimization of the joint-height differences for a sample free-form architectural design that is meshed with a quad-dominant mesh.
\end{abstract}

Keywords: Planar Mesh; Support Structure; Post-Optimization; Height-Difference Function

\section{Introduction}

In the physical realization of a project the arbitrary shapes designed by the architect must satisfy a number of requirements that limit the possible realizable solutions for the final "free forms". Those are frequently used in "sculptured" designs, like museums and towers that are intended to be city landmarks. Such designs seek their representation through shape, while applying cost-effective materials and making use of the whole of the available area.

The freeform surfaces of the structures can be described by different types of meshes providing the planarity of each of the mesh surface elements, i.e., the faces. The existing freeform structures mainly consist of traingular meshes, where the condition of planarity is satisfied automatically. We chose to use quadrilateral meshes [1] (Figure 1), because they are cost effective, but also geometrically complex. The condition of planarity is satisfied by an optimization algorithm based on Sequential Quadratic Optimization [2].

Freeform façades consist of relatively thick, planar (Figure 2), panel elements and of a corresponding support structure. The outer surfaces of the panel elements coincide with the mesh faces (Figure 3). To ensure the contact of the edges (Figure 3) of the neighboring panel

\footnotetext{
*Post-optimization of height differences of Freeform Structures.
}

elements along their thicknesses, their edges must be cut at different angles, which causes differences in the edge heights and furthermore differences in the positions of the inner metal sheets of the insulation panels.

In common support structures (Figure 4) where the beams have the same height, the position of the beams must compensate for the above-mentioned difference in the positions of the outer metal sheets. For this reason relatively large joint-height differences $\Delta h_{i, j}$ (Figures 57) are necessary.

The differences in the positions of the beams ensure a

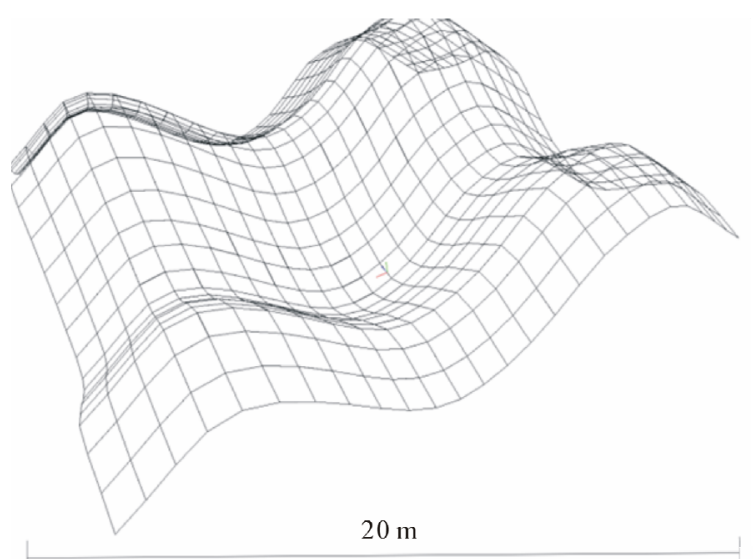

Figure 1. Double-curved freeform quad-dominant mesh. 


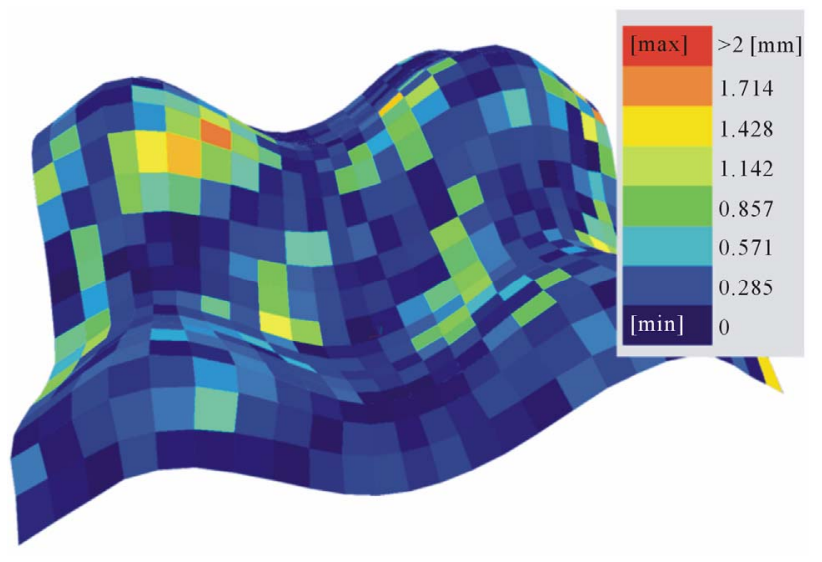

Figure 2. Planar optimized mesh.

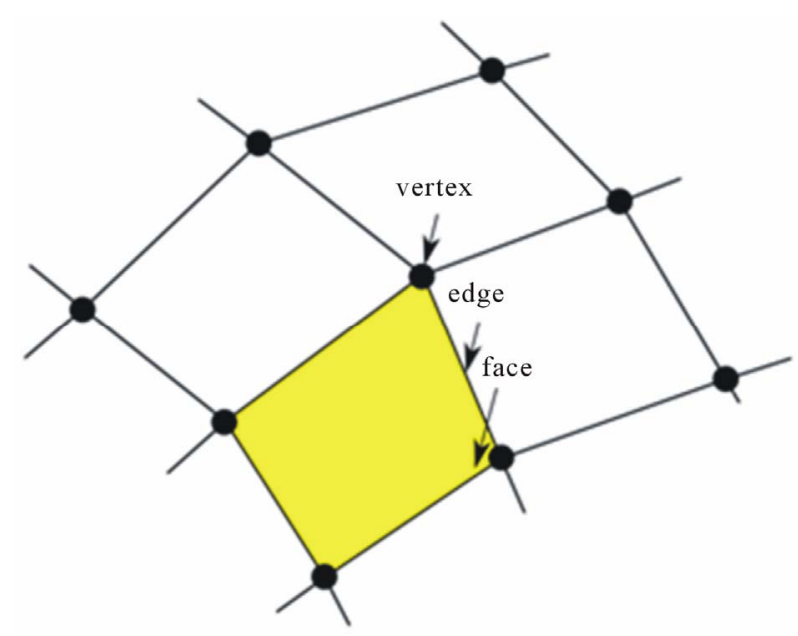

Figure 3. Mesh elements: vertex, edge and face.

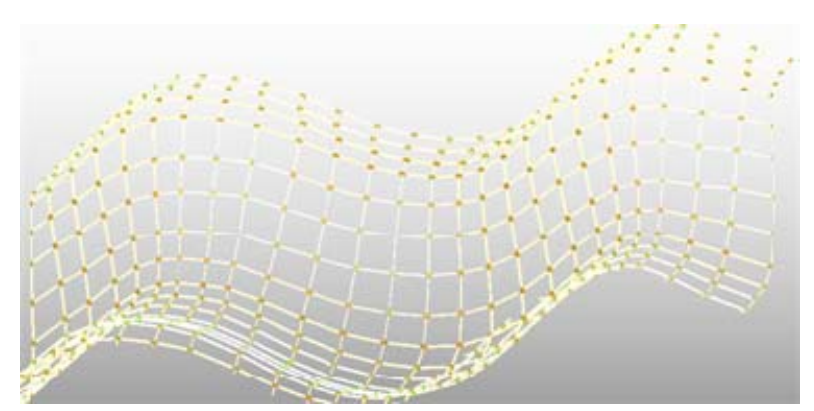

Figure 4. CAD model of a support structure of planar mesh structure.

constant distance between the outer metal sheet and the beams of the structure. Producing, as a result, the different vertex height distances $h_{i, j}$ (Figure 6).

The aim of this research is to develop a post-optimization procedure by which the minimum joint-height differences $\Delta h_{i, j}=h_{i, j ; \max }-h_{i, j ; \text { min }}$ can be achieved for all the joints, taking into account all the faces of the considered free-form architectural design. It should be pointed out that the angles $\alpha_{i, 1}$ and $\alpha_{i, 2}$ in all the joints are already optimized [3,4] (Figures 6 and 7) and therefore not subject to change.

Freeform structures require planarity for each closure metal panel (insulation panel element) [5-7]. Planarity is necessary for the building of the structure, particularly in cases when the structure is covered with non-deformable elements (e.g., glass). We are trying to make planar elements for the selected structure, while still keeping the

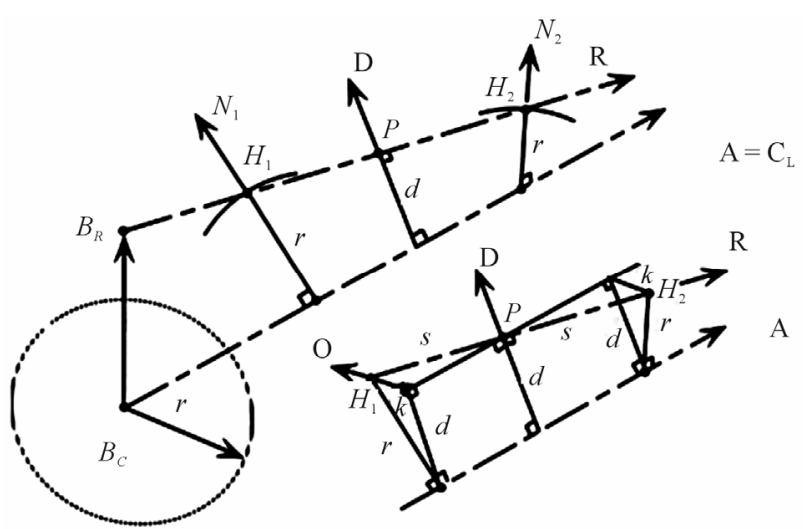

Figure 5. Geometry of the cylinder-beam intersection problem.

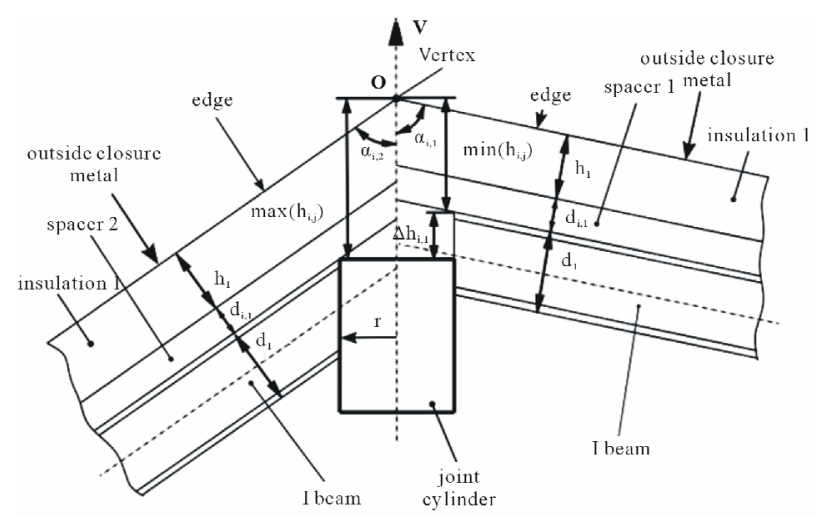

Figure 6. Initial vertex cross-section with equal spacer thickness $\left(h_{2}+d_{i, 2}\right)$.

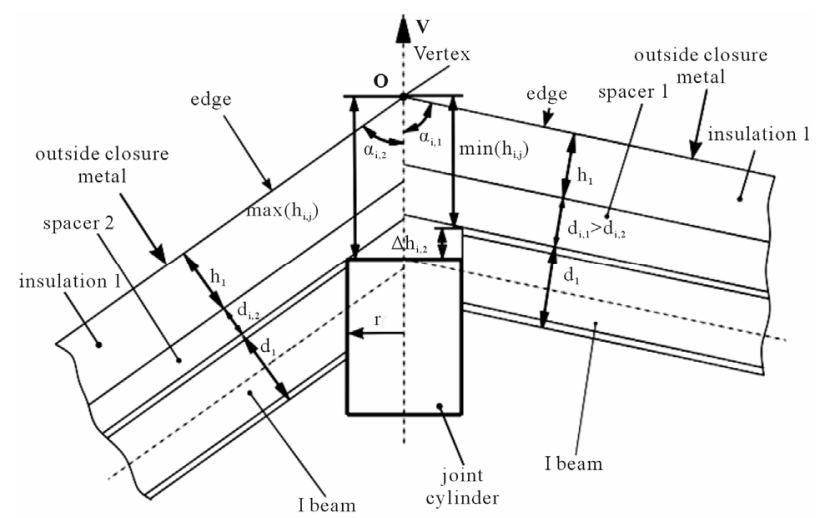

Figure 7. Post-optimized vertex cross-section with variable spacer thickness $\left(\Delta h_{i, z}<\Delta h_{i, 2} \rightarrow d_{i, z}<d_{i, 2}\right)$. 
original outside form of the structure, as designed by the architect. Triangular mesh elements do not require planarization because their geometry is always planar. The planarity of an element in a selected mesh should be executed to the level that still allows the assembly of closure elements.

\section{Problem Formulation}

Figure 4 shows a CAD model, designed according to planar and conical pre-optimized mesh. Figure 4 shows the support structure only; composed of joint cylinders and I beams. In every single joint cylinder (or joint box [8]) is positioned according to a maximum vertex height distance $\left(h_{i, j ; \max }\right)$. Therefore the top flange of the corresponding I beam is leveled with the top edge of the joint cylinder. All the other I beams, having smaller vertex height distances, must be positioned higher, producing additional internal loading in the joint cylinder and joint cylinder-beam connection problems. The idea is to provide minimal possible joint height differences $\left(\Delta h_{i, j}\right)$, between top flange of the I beams in every single joint cylinder, which significantly reduces additional forces and moments in a joint cylinder (Figure 7). An postoptimization algorithm was created to do the task for all the joints in the structure. In chapter 4, a graphical analysis of joint height differences is made for the entire mesh of the sample free form structure. Minimization of these differences in particular joint significantly reduces additional forces and moments in a joint cylinders.

\section{Related Work}

Not many papers cover our ideas presented in the introduction. Multi-layer architecture, including planar and conical meshes was discussed by Pottmann et al. [2, $5,9,10]$. Although visually appealing P-hex meshes that were also extended to meshes with parallel edges. P-hex geometry inherits similar problems with a physical realization of vertex. The elimination of edge offset differences can be achieved with Koebe polyhedra [11], but this brings very restrictive geometry, which cannot approximate arbitrary shapes. Still this is a promising approach for glass structures with no closure layer provided. Such surfaces can also be part of the freeform structure that can be included in mesh optimization as a rigid body. Pottmann et al. [10] also suggest to approximate beam offsets with fairness functional during vertex perturbation. In their optimization they neglect the physical realization of the vertex junction and concentrate on optimization to achieve an approximately constant offset from a theoretical point of view. So far, we are unaware of any architectural project that should use the present geometry processing ideas, as it seems that solutions need to be solved in detail in CAD (Figure 4) before the realization is possible. In addition, a stress analysis of such structure is required, which is not a trivial task as adequate stucture computations are yet to be determined.

\section{Post-Optimization Method}

\subsection{The Joint Connection Differencesat Cylinder}

In this section we briefly present the geometric algorithm to locate the intersection points between a ray and a cylinder following the Cychosz and Waggenspack [12] algorithm.

We are only interested in the intersection point $H_{\text {in }}$ between the vertex cylinder and the beam. Connecting height $d$ (Figure 5) from vertex origin $\boldsymbol{o}$ and beamcylinder intersection $H_{i n}$ is calculated by projecting vector $H_{i n}-\boldsymbol{o}$ to vertex normal $\boldsymbol{v}$ (see Figure 6). After some algebra calculations we finally obtain

$$
d=\left|\boldsymbol{o}-H_{\text {in }}\right| \frac{\boldsymbol{v} \cdot\left(\boldsymbol{o}-H_{\text {in }}\right)}{|\boldsymbol{v}|\left|\boldsymbol{o}-H_{\text {in }}\right|}
$$

Beam offset distance $d$ can be regarded as a function of many parameters, face normals, inclination and di hedral angles. This function is obviously nonlinear and so is the post-optimization procedure that generally minimizes differences between each beam $d$ in each vertex of the structure. Solving such global post-optimization problem can easily lead to local optimums. Especially when structures are large. Many algorithms from computational geometry try to avoid such situations by defining local operators rather than solving the problem globally. In this paper we follow such ideas by introducing two competitive algorithms with local impact and compare their results.

\subsection{Vertex Element Differences}

In order to generate a CAD model, it is necessary to specify the points where the support structures and the joint elements are positioned. The beams are displaced at the fixing points in the joint cylinder. The calculation of the beam positions, displaced from the reference points, is shown below.

Figure 6 shows a cross-section of the cylindrical joint, beams with insulation, spacers and outside closure metal. The outside closure sheet normals $\boldsymbol{n}_{1}$ and $\boldsymbol{n}_{\mathbf{2}}$ are joined at the vertex reference point $o$ (Figure 6).

$$
h_{i, j}=h_{1} \cdot \frac{\boldsymbol{n}_{\boldsymbol{e}} \cdot \boldsymbol{n}_{\mathbf{1}}}{\left\|\boldsymbol{n}_{\boldsymbol{e}}\right\| \cdot\left|\boldsymbol{n}_{\mathbf{1}}\right|}+\left|r_{e} \cdot \boldsymbol{e}-\boldsymbol{r}\right|
$$

where $\boldsymbol{n}_{\boldsymbol{e}}=\frac{\boldsymbol{n}_{\mathbf{1}} \times \boldsymbol{n}_{\mathbf{2}}}{\left|\boldsymbol{n}_{\mathbf{1}} \cdot \boldsymbol{n}_{\mathbf{2}}\right|}$ and $r_{e}=r \cdot \frac{|\boldsymbol{e}|\left|\boldsymbol{n}_{\boldsymbol{e}}\right|}{\boldsymbol{e} \times\left(-\boldsymbol{n}_{\boldsymbol{e}}\right)}$.

Vertex positioning. For each vertex $i$ of the selected 
mesh, the vertex height distance $h_{i, j}$ is determined:

$$
h_{i, j ; \max }=\max \left(h_{1,1}+d, \cdots, h_{1, i}+d, \cdots, h_{1, n}+d\right)
$$

where the index $i=1 \cdots n$ represents the vertices, and the index $j=1 \cdots m$ represents the beams of a given mesh.

\subsection{Definition of the Height-Difference Function}

The height-difference function is created by taking into account all the vertex height differences in the selected mesh.

$$
F(h)=\sum_{i=1}^{n} \sum_{j=1}^{m}\left(\left|h_{i, j}-h_{i, j+1}\right|+\left|h_{i, j}-h_{i+1, j}\right|\right)
$$

where $i=1 \cdots n$ and $j=1 \cdots m$.

The index $i$ represents the vertices; the index $j$ denotes the $I$ beams.

The first part of the height-difference function represents the vertex height differences $h_{i, j}$ (see Figure 6), while the second part of the height-difference function represents the connection of the current vertex with the neighboring vertices. The height-difference function compares the vertex height differences of the support structure on both sides of the beam.

\section{Results}

The resulting shift of the beams introduces different distances between the outside closure metal of the panel and the individual beam (see $d_{i, 1}$ and $d_{i, 2}$ in Figure 7). These differences are compensated for by the spacers of adapted thicknesses (Figures 6 and 7). After optimization the thickness $d_{i, 1}$ is no longer a constant for the whole of the façade, but can be different for each beam, $\Delta h_{i, 2}<\Delta h_{i, 1} \rightarrow d_{i, 2}<d_{i, 1}$ (see Figure 7).

Figure 8 shows the convergence of the height-difference function (Equation (4) that describes the problem of joint-height differences $\Delta h_{i, j}$ (Figures 6 and 7), which we would like to minimize. The height-difference function is composed of the sum of the vertex height differences $\left(h_{i, j}\right)$ for each vertex in a given mesh. The constraint, determining the interval by which each beam can move, is $[-5,30] \mathrm{mm}$.

In ten iterations, the height-difference function reaches a maximum drop, followed by reaching almost the top convergence after 15 iterations and not changing significantly up to the end.

A comparison between the individual vertex height distances for the beams before and after optimization is shown as a standard deviation in Figure 9. It shows the standard deviation of the vertex height differences $h_{i, j}$ (Figure 6) of the beams for all the vertices of the mesh. It can be seen that the deviation of the vertex height differences $h_{i, j}$ (Figures 6 and 7) is reduced.

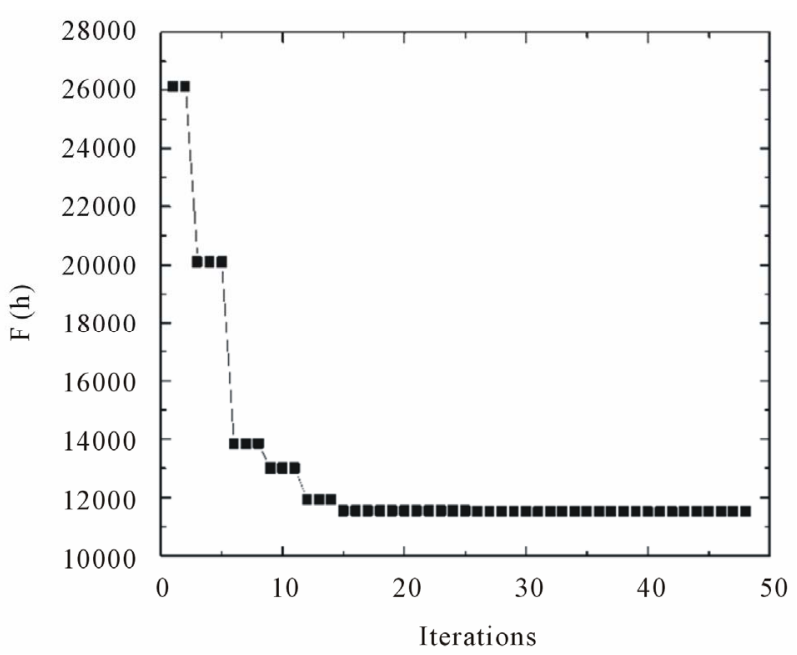

Figure 8. Height-difference function [mm] convergence.

Figure 9 shows that the standard deviation of the vertex height distances from the vertex improves in almost all the vertices after optimization. On the vertices with only three beams, which are mostly on beam vertices, it is not possible to achieve a quasi-ideal situation.

It can be concluded that the distribution of the jointheight distances $\left(\Delta h_{i, j}\right)$ on the mesh is improved after the post-optimization procedure. This post-optimization procedure results in more constant joint-height distances on the mesh. We believe that this optimized mesh is: 1) cost effective, and 2) simpler to construct.

\section{Conclusions}

The aim of the post-optimization procedure is to provide the minimum joint-height differences $\Delta h_{i, 2}<\Delta h_{i, 1}$ (Figure 7). The focus is on quadrilateral meshes because they are cost effective. An analysis of the initial and optimized meshes was made in order to ascertain whether the structure is improved after the optimization and whether the mesh keeps its original shape.

The first part is represented by the post-optimization algorithm. It provides, globally, the minimum jointheight differences for the beams in the individual vertices of a given mesh (Figure 1). The optimization tries to provide the minimum joint-height distances $\left(\Delta h_{i, j}\right)$, which provides the strength stability (the additional forces and momentums in the joint cylinder are minimal) of the construction. The height-difference function for the optimization is so structured that it keeps the original shape of any given mesh (Equation (4)).

The second part involves a mesh analysis before and after the optimization. In a quasi-ideal situation the standard deviation of the vertices would be zero (Figure 8), which would mean that all the vertex's beams have the minimum joint-height differences. We believe that this optimized mesh is: 1) cost effective, and 2) simpler to 


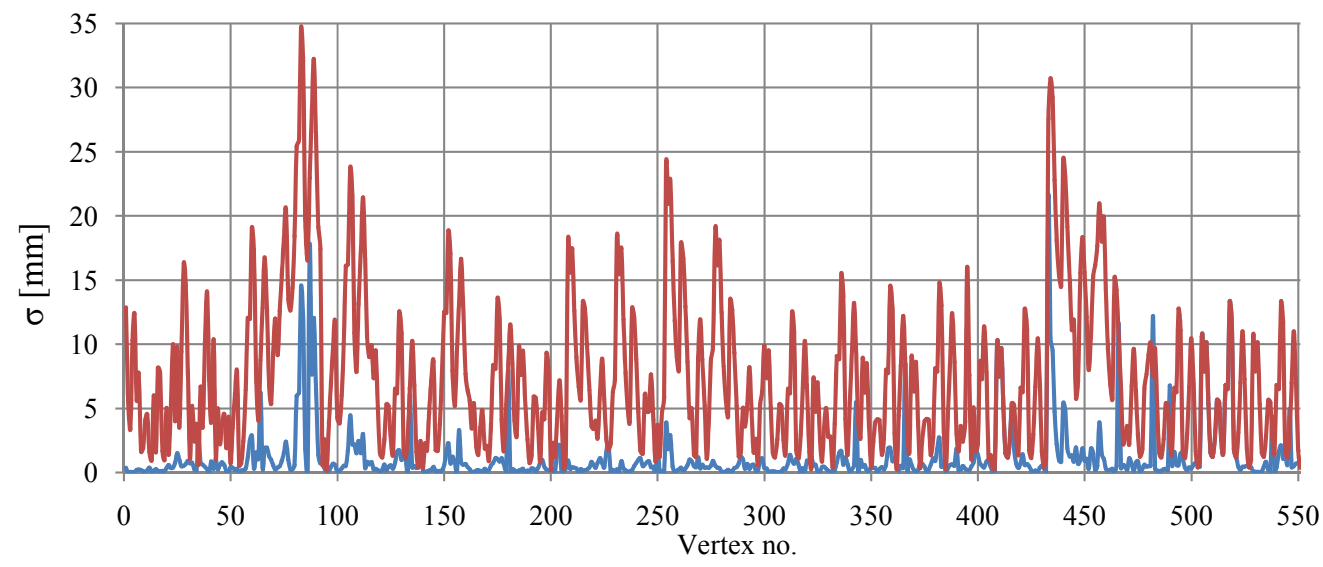

Figure 9. Standard deviation of vertex height distances before and after post-optimization.

construct.

\section{REFERENCES}

[1] S. Kulovec, L. Kos and J. Duhovnik, "Mesh Smoothing with Global Optimization under Constraints," Strojniškivestnik Journal of Mechanical Engineering, Vol. 57, No. 7, 2010, pp. 555-567.

[2] H. Pottmann and J. Wallner, "The Focal Geometry of Circular and Conical Meshes," Advances in Computational Mathematics, Vol. 29, No. 3, 2008, pp. 249-268. doi:10.1007/s10444-007-9045-4

[3] L. Kos, S. Kulovec, V. Zaletelj and J. Duhovnik, "Structure Generation for Free-Form Architectural Design," Advanced Engineering, Vol. 3, No. 2, 2009, pp. 187-194.

[4] L. Kos, S. Kulovec and J. Duhovnik, "Support Structure Optimization for Freeform Architectural Design," TMCE 2010, University of Technology, Delft, 2010, pp. 829840.

[5] W. Wang, Y. Liu, D. Yan, B. Chan, R. Ling and F. Sun, "Hexagonal Meshes with Planar Faces," Tech. Rep. TR2008-13, Department of Computer Science, The University of Hong Kong, Hong Kong, 2008.
[6] K. Madsen, H. B. Nielsen and O. Tingleff, "Optimization with Constraints," 2nd Edition, 2004.

[7] O. Schenk and K. Gärtner, "Solvingunsymmetric Sparse Systems of Linear Equations with Pardiso," Journal of Future Generation Computer Systems, Vol. 20, No. 4, 2004, pp. 475-487. doi:10.1016/j.future.2003.07.011

[8] J. Duhovnik, S. Kulovec and L. Kos, "Multiple Vertex Joint Adapter," Patent No. p201000325, 2010.

[9] H. Pottman, A. Asperl, M. Hofer and A. Kilian, Eds., "Arhitectural Geometry," Bentley Institute Press, Exton, 2008.

[10] H. Pottmann, Y. Liu, J. Wallner, A. Bobenko and W. Wang, "Geometry of Multi-Layer Freeform Structures for Architecture," ACM Transactions on Graphics, Vol. 26, No. 3, 2007, Article No. 65. doi: $10.1145 / 1276377.1276458$

[11] A. I. Bobenko, T. Hoffmann and B. A. Springhorn, "Minimal Surfaces from Cicle Patterns: Geometry from Combinatorics," Annals of Mathematics, Vol. 164, No. 1, 2006, pp. 231-264. doi:10.4007/annals.2006.164.231

[12] J. Cychosz and W. Waggenspack Jr., "Intersecting a Ray with a Cylinder," Academic Press, London, Vol. 2, 1994, pp. 353-365. 